\title{
Location Privacy in Wireless Personal Area Networks
}

\author{
Dave Singelée \\ ESAT-COSIC \\ Katholieke Universiteit Leuven \\ Heverlee, Belgium \\ Dave.Singelee@esat.kuleuven.be
}

\author{
Bart Preneel \\ ESAT-COSIC \\ Katholieke Universiteit Leuven \\ Heverlee, Belgium \\ Bart.Preneel@esat.kuleuven.be
}

\begin{abstract}
Location privacy is one of the major security problems in a Wireless Personal Area Network (WPAN). By eavesdropping on the transmitted packets, an attacker can keep track of the place and time of the communication between the mobile devices. The hardware address of the device can often be linked to the identity of the user operating the mobile device; this represents a violation of the user's privacy. Fortunately, this problem can be solved quite efficiently in a WPAN. We consider four communication scenarios and present several techniques to solve the location privacy problem in each of these scenarios. As mobile devices in a WPAN are typically operated by a user and energy constrained, we focused on user-friendliness and energy consumption during the design of our solutions.
\end{abstract}

\section{Categories and Subject Descriptors}

C.2.0 [Computer-Communication Networks]: GeneralSecurity and protection

\section{General Terms}

Algorithms, Security

\section{Keywords}

Location privacy, Bluetooth, pseudonyms

\section{INTRODUCTION}

\subsection{Wireless Personal Area Networks}

During the last couple of years, there is a strong tendency towards mobility for IT and computer networks. Wireless networks are rapidly gaining in importance; after all, wiring collides with the increasing demand for mobility. This paper focusses on Wireless Personal Area Networks (WPAN). A WPAN is a small, heterogeneous, wireless ad-hoc network used for communication among several personal mobile devices. For example, a WPAN can consist of a PDA, a mobile

Permission to make digital or hard copies of all or part of this work for personal or classroom use is granted without fee provided that copies are not made or distributed for profit or commercial advantage and that copies bear this notice and the full citation on the first page. To copy otherwise, to republish, to post on servers or to redistribute to lists, requires prior specific permission and/or a fee.

WiSe'06, September 29, 2006, Los Angeles, California, USA

Copyright 2006 ACM 1-59593-557-6/06/0009 ...\$5.00. phone, a portable computer, a wireless headset, ... These devices often belong to the same user. The range of a WPAN is typically a few meters. A WPAN can be realized with network technologies such as IrDA [13] and Bluetooth [4]. In this paper, we focus on Bluetooth, but the concept of our technical solutions can also be applied to all other types of Wireless Personal Area Networks.

The Bluetooth wireless technology $[3,4,9]$ realizes a lowcost, short-range, wireless voice- and data-connection through radio propagation. It operates on the media access control layer. With a normal antenna, the maximal range is about $10 \mathrm{~m}$. The Bluetooth wireless technology uses the $2.4 \mathrm{GHz}$ band, which is unlicensed, and can be used by many other types of devices such as cordless phones, microwave ovens, WiFi [25] and baby monitors. Every time a Bluetooth wireless link is formed, it is within the context of a piconet. A piconet consists of at most 8 devices that occupy the same physical channel. In each piconet, there is exactly one master, the other devices are called slaves. In June 2000, the Bluetooth standard was included in IEEE 802.15 [12], the Wireless Personal Area Network Working Group.

It is possible to configure the "visibility" of a Bluetooth device. When a device is in non-discoverable mode, it does not respond to inquiries of other devices. When the device is in limited discoverable mode, it is discoverable only for a limited period of time, during temporary conditions or for a specific event. And finally, when it is in general discoverable mode, it is discoverable (visible) continuously. Each device is characterized by a factory-established 48-bit identifier, unique for every device: the Bluetooth hardware address.

\subsection{Location Privacy}

The Bluetooth standard also describes the security protocols of the Bluetooth security architecture for Wireless Personal Area Networks. The most critical part of this security architecture is without doubt the key agreement protocol. However, several security flaws have been identified within the Bluetooth protocols [14,23]. E.g., all keys depend on a low-entropy shared secret (the PIN) and there are some Denial of Service attacks that are very easy to perform.

Another crucial problem is location privacy. When two or more Bluetooth devices are communicating, the transmitted packets always contain the Bluetooth hardware address of the sender and the destination (or an identifier which is directly related to this address). When an attacker eavesdrops on the transmitted data, (s)he knows the Bluetooth hardware addresses of these devices. The attacker does not have to be physically close to the communicating devices, 
(s)he can use a device with a stronger antenna (e.g., it is very easy to construct an antenna which can intercept Bluetooth communication from more than one mile away $[5,6]$ ) or just place a small tracking device near the two Bluetooth devices. Even devices that are in non-discoverable and nonconnectable mode are vulnerable to location privacy attacks. Wong and Stajano [26] demonstrate that one can recover the Bluetooth hardware address via the frequency hop pattern of the device. This attack only requires a few packets and a work factor of $2^{28}$.

By observing the Bluetooth hardware addresses, the attacker can keep track of the place and time mobile devices were communicating, which corresponds to a violation of the privacy of the user. This is a very important problem in mobile networks. E.g., without location privacy, a terrorist could be capable of discovering in which hotel (and even in which room) an important politician stays. Suppose one would be able to continuously track the movements of the president of the United States. This would certainly entail serious security problems. Another example of an attack is to track users on a specific location and use this information for location dependent commercial advertisements (e.g., a shop can send advertisements to everybody that is nearby). These attack cases illustrate that it should be possible for the user to decide when his/her location is revealed and when not.

Tracking mobile devices is not a theoretical problem. Several practical implementations have already been demonstrated. A Bluetooth positioning and tracking application was first implemented in the Aalborg Zoo, Denmark's largest zoological garden. Special "Bluetags" [2] were given to the visitors. These are special body tags that have to be pinned to children, which allow parents to position and track the movement of their child within the Zoo, and this is very effective to trace lost children. This solution is an example of a beneficial use of the Bluetooth technology. The idea of using Bluetooth in positioning and tracking can also have a dark side. E.g., it can be used for the surveillance of citizens within a city. The perfect example of such a system exists as the Loca project [15]. It consists of small network of Bluetooth receivers deployed in public spaces within the Helsinki city, connected to the central Loca server via GPRS. The receivers report the position of any Bluetooth devices within proximity to the server, which then can send a message to the Bluetooth device such as "you were at the market place yesterday at $22 \mathrm{~h} 03 . "$

This paper proposes several techniques to solve the location privacy problem. Mobile devices in a WPAN can communicate in different ways to each other, depending on the information (e.g., secret keys) that they already share. A classification into different communication scenarios can be made. We show how to ensure location privacy in each of these scenarios.

\subsection{Organization of the paper}

In the previous section, an introduction to the concept of location privacy was presented. A classification into four different WPAN communication scenarios is made in Sect. 2. Location privacy can be enabled in each of these scenarios. This is conceptually demonstrated in Sect. 3. In practice, different scenarios will occur at the same time and this has some important consequences This will be discussed in Sect. 4. Finally, Sect. 5 concludes this paper.

\section{OVERVIEW OF WPAN SCENARIOS}

Let us first clearly define the problem we are going to focus on. There are two mobile devices, called $A$ and $B$, that want to communicate privately. We implicitly assume that both devices are personal devices, belonging to a specific user (this does not have to be the same user). If the devices cannot be linked to a user, location privacy would not really be an issue. In the rest of this paper, we assume that $A$ initiates the communication. $A$ sends a message to $B$ using a wireless communication technology (e.g., Bluetooth). Such a message consists of a header and a payload. The header contains identification information (typically the address of the sender and receiver or information that is directly related to these addresses), the payload just plain data (encrypted or not). As Bluetooth operates on the media access control layer, we will only focus on this layer, and not on the layers above.

This paper investigates how $A$ can send a message to $B$, in such a way that $B$ still knows the message was intended for him, but that an attacker has no information about the identity of $A$ and $B$. We assume that the attacker, which is computationally bounded, does not only passively eavesdrop on the data, but is also able to perform active attacks such as replay attacks or inserting dummy traffic. The goal of our solutions is to establish location privacy in the presence of such an active eavesdropper. More precisely, we want to prevent two types of attacks:

- It should be computationally hard for an attacker, who observes the exchanged messages, to detect which specific device is participating in the communication. This property is called untraceability. Note that it is not a problem that an attacker detects a device is sending and/or receiving data, and that the attacker is even allowed to know the precise location of this device (e.g., by observing the signal strength of the radio transmission). However, the attacker should not be able to determine the exact identity of this device. We implicitly assume that the attacker has no prior knowledge about which devices are communicating. Otherwise, privacy problems would occur in scenario 2 (see Sect. 3.2).

- It should be computationally hard for an attacker to link several messages to one sender and/or receiver (even without knowing the exact identity of this device). This property is called unlinkability. If one can detect when a certain (unknown) device is communicating, one could maybe use this information to discover the real Bluetooth hardware address of the device (e.g., by observing certain specific communication patterns) and hence track it.

Note that one can not make use of a central trusted server in a WPAN, as it is typically a short-range, dynamic ad-hoc network. So traditional pseudonym systems [16] cannot be used. The mobile devices themselves have to make sure that location privacy is ensured. They will use shared data to compute a common identifier that replaces the identification information in the header of the message. This random identifier, which certainly has to be variable, will appear as random data for an eavesdropper, but $B$ will recognize it and hence know the message was intended for him. The way the common identifier is computed depends on the data 


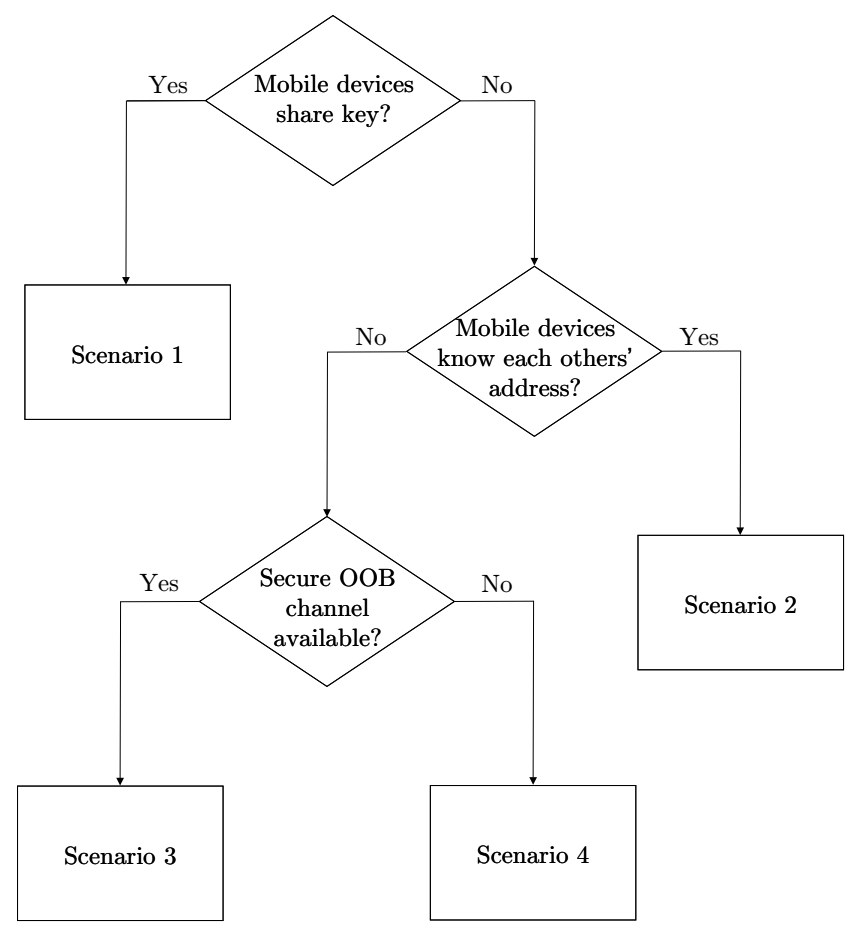

Figure 1: Our 4 WPAN scenarios

the mobile devices share. We envision four communication scenarios, as depicted in Fig. 1:

- Scenario 1: the mobile devices share a symmetric key. This key can be the result of a key establishment protocol during a secure initialization phase. It can also be a session key that has been used before to communicate securely with each other. For efficiency reasons, we only consider symmetric keys, as public key cryptography is far more energy consuming [20]. It is however possible to transform our solution into a public key variant.

- Scenario 2: $A$ knows the address of $B$. This address could have been entered by a user, or $B$ could have sent it during a secure inquiry (or initialization) phase. Another possibility is that $A$ knows this address from previous communication rounds. Note that the only conceptual distinction between an attacker and device $A$, is that the former has no prior knowledge about the address of $B$.

- Scenario 3: a secure extra communication channel is available. This out-of-band channel can be private and/or authentic. Typical examples of outof-band channels are infrared (IrDA [13]), physically connecting both devices [24], the user operating the device, a verbal side channel, ...

- Scenario 4: the mobile devices share nothing. They do not have communicated before or did not store any information from previous communication rounds (e.g., keying material). The devices also do not know each others' addresses.
In the next section, a solution for each scenario is presented. We especially focus on user-friendliness and the energy cost. Enabling location privacy should only cause a minor increase of the total energy consumption, as mobile devices in a WPAN are typically energy constrained.

\section{ENABLING LOCATION PRIVACY}

It should be possible for the user to decide when his/her location is revealed and when not. When (s)he wants to enable location privacy, (s)he will use one of the solutions described in this section. The most appropriate technique depends on the communication scenario (see Sect. 2 for an overview).

\subsection{Scenario 1: Mobile devices share a sym- metric key}

In this scenario, we assume that $A$ and $B$ share a symmetric key $k$ (e.g., established during a secure initialization phase). We will not focus on the cryptographic methods to establish such a key, this is out of the scope of this paper. For efficiency reasons, we do not consider public key cryptography, but the concept remains the same.

Every mobile device has in its memory a list of symmetric keys it shares with the other devices. For every key it shares, it computes an identifier $\mathrm{R}$ as follows:

$$
R=P R F_{k}(I V)
$$

$P R F$ is a pseudo-random function ${ }^{1}$ (with an output size of $48 \mathrm{bits}$ ), $k$ the shared key (length of 48 bits is sufficient), and $I V$ a random, publicly known 48-bit value. A good choice for a pseudo-random function is a Message Authentication Code $(M A C)$. See [19] for an overview of recommended secure Message Authentication Codes. It is important that all devices in a WPAN use the same $I V$ (e.g., one could agree to always use the value $00 \ldots 01$ ). These identifiers $R$ are stored in memory. When $A$ wants to send a message to $B$ for the first time, it puts the corresponding identifier $R$ in the header of the message (in the address field of the destination and the sender). $B$ will recognize the identifier $R$ and hence will know the message was intended for him. Next, both devices update their identifier $R$ as follows:

$$
R_{\text {new }}=P R F_{k}\left(R_{o l d}\right)
$$

This way, a chain of pseudo-random (e.g., MAC) values is computed. Updating $R$ every message is necessary to avoid tracking. All consecutive messages will contain different identifiers $R$, and only a device that knows $k$ can link the related identifiers to each other, or calculate the next value in the chain. An eavesdropper just sees random values $R$ and has no idea which devices are communicating with each other. An attacker can not even verify if a specific device is participating in the communication. As we will show later, this property is not valid anymore in the other scenarios. This technique is the most secure one described in this paper. That is why we recommend to use this solution when the two Bluetooth devices share a symmetric key $k$ and also know each others' addresses.

A device does not have to wait until it receives a message to compute the next identifier $R$, it can calculate the entire chain in beforehand. However, if the mobile device is

\footnotetext{
${ }^{1} \mathrm{~A}$ computationally bounded attacker can not distinguish the output of a pseudo-random from a random bitstring.
} 
memory constrained, then it should only store the current identifier $R$. Data storage is hence not a problem. One has to take into account that some messages will not be received properly, due to communication errors. To avoid that one device would update $R$ and the other device wouldn't, one should only discard old values of the chain when one receives a correct message from the other device. When the identifier $R$ of the message is not the next value in the chain, but an older value, one knows that the other device did not receive some of the previous messages. When the other device uses an identifier $R$ which is already further ahead in the chain, one knows that one did not receive some messages and one could ask for retransmission. This way, the devices will stay synchronized. Of course, one could also employ a different update policy.

An alternative solution would be to use a counter instead of the old identifier $R$ as input for the pseudo-random function, as proposed by Wong and Stajano [26]. This is however completely equivalent to the solution described above.

A similar solution [8] has been proposed to protect the location privacy of 48-bit WiFi addresses in a Wireless LAN (WLAN). The authors use MD5 hashes, started with an unpredictable random seed, to construct temporary pseudonyms (the last hash constitutes the input for the next application of the hash function). This solution can however not be deployed in this scenario. Unlike WLAN, Bluetooth does not employ a client/server topology. As Bluetooth devices communicates with different devices in the same time (point to multipoint communication), a keyed pseudo-random function is needed instead of a hash function.

Our solution based on a chain of pseudo-random values is also very efficient. Suppose one uses the HMAC algorithm to calculate the chain. Experiments show that this HMAC function consumes $1.16 \mu \mathrm{J} /$ Byte on an Intel SA-1110 StrongARM processor clocked at $206 \mathrm{MHz}$ [20]. As our input is 6 bytes, our technique introduces an extra energy cost of 6.96 $\mu \mathrm{J}$. This is completely negligible to the cost of transmitting or receiving a 48-bit identifier, which is $1007 \mu \mathrm{J}$ and $670 \mu \mathrm{J}$ respectively.

\subsection{Scenario 2: Address known by other mo- bile device}

In this scenario, we assume that $A$ knows the address of $B$, the device it wants to send a message to. This address could have been entered by a user, or $B$ could have sent it during a secure inquiry (or initialization) phase. Another possibility is that $A$ knows this address from previous communication rounds. However, both devices do not share a (symmetric) key. Otherwise, the solution described in the previous scenario should be employed. We will not use Identity Based Cryptography (IBC) [21], as this technique consumes too much energy. As in the previous scenario, we want to put an identifier $R_{B}$ in the header of the message, so that $B$ knows the message was intended for him. To avoid tracking, the identifier $R_{B}$ has to be different in every message, and it should be computationally hard for an attacker, who does not know the address of $B$, to link a specific identifier $R_{B}$ to device $B$. When $A$ wants to send a message to $B$, it computes $R_{B}$ as follows:

$$
R_{B}=H(\operatorname{addr} B, \text { nonce })
$$

$H$ is a cryptographic ${ }^{2}$ hash algorithm (one only needs 48 ${ }^{2}$ A cryptographic hash function should be preimage and sec- bits of the output), $a d d r B$ the Bluetooth hardware address of $B$ and nonce a random value. For security reasons, the length of the nonce should be at least the same as the length of the address (48 bits in the case of Bluetooth).

To avoid tracking, the nonce should be different in every message that is sent to the same mobile device. Otherwise, one would reuse the same identifier $R_{B}$ and an attacker would detect that both messages are sent to the same device. There are several methods to ensure that the nonce is random and variable:

- One could assign 48 bits of the payload to the nonce. This is the most efficient solution, but one has to be careful. As several messages often will start with the same bit sequence (e.g., some bits that indicate the application that is running on top of Bluetooth), it would be best not to use the first bits of the payload to generate the nonce. Otherwise, the nonce would not be variable and tracking would still be possible. If the payload is encrypted, then it is perfectly safe to use the first 48 bits of the message.

- Instead of assigning 48 bits of the payload to the nonce, one could take the entire payload as the nonce. After all, there is no maximum length of the nonce. This solution is more energy consuming than just using 48 bits, but it increases the probability of the nonce being variable. One would normally not send exactly the same message twice to the same mobile device.

- One could also generate a random 48-bit number (the nonce) and use the first 48 bits of the payload to transmit this random nonce. This way, the probability of reusing the same nonce is very low. It is however by far the most expensive solution, as one can send fewer bits of real data (in the case of Bluetooth, one would lose 48 of the 2745 bits of the payload).

- Another solution would be to assign the value of a counter to the nonce. Every time a message is sent, the counter is incremented. Of course, one can not include the value of the counter in the message, as this would enable an attacker to link messages. Assigning the value of a counter to the nonce has several drawbacks. Both devices have to keep the counter synchronized, otherwise communication will fail. Another disadvantage is that the device has to store the counter of every device it is communicating with. This results in a major storage cost. That is why we do not recommend this solution.

For every message that $B$ receives, it computes the hash value of its own address and the nonce. If this value corresponds to the identifier $R_{B}$ in the header of the message, then $B$ knows the message was intended for him. If the value does not corresponds, $B$ has no information about the correct destination of the message.

It is computationally very hard for an attacker, who does not know the address of $B$, to link the identifier $R_{B}$ to the

ond preimage resistant (given $\mathrm{h}$, it should be hard to find any $\mathrm{m}$ such that $h=\operatorname{hash}(m)$ or $h=\operatorname{hash}(\operatorname{hash}(m)))$, and collision-resistant (it should be hard to find two different messages $m 1$ and $m 2$ such that hash $(m 1)=$ hash $(m 2))$. See [19] for an overview of recommended cryptographic hash functions. 
correct device. (S)he would have to perform a brute force attack, and try all possible 48-bit addresses to find the correct one. However, an attacker can still track a specific device, from whom (s)he knows the hardware address. This can simply be done by checking if the hash value of this specific address and the nonce corresponds to the identifier $R_{B}$. This attack can not be avoided, as the only difference between an attacker and $A$ is the knowledge of the address of the destination $(B)$. The attack is however not possible in scenario 1 (see Sect. 3.1), as an eavesdropper does not know the secret key.

The header of a message does not only contain an address field of the destination (which encloses the identifier $R_{B}$ ), it also embodies the address field of the sender. Of course, $A$ does not put its Bluetooth hardware address in this field. Instead, it generates a random identifier $R_{A}$ (with a bit length of 48 bits) and inserts this in the address field. How does $B$ reply to the message of $A$ ? It first computes an identifier $R_{\text {reply }}$ as follows:

$$
R_{\text {reply }}=H\left(R_{A}, \text { addr } B, \text { nonce }\right)
$$

$H$ is a cryptographic hash algorithm, $R_{A}$ the random identifier of the destination $(A), \operatorname{addr} B$ the Bluetooth hardware address of the sender $(B)$ and nonce a random value. The nonce should be variable and hence different from the nonce used in eqn. 3. In order to ensure that the nonce is random and variable, one could use one of the methods described earlier in this section. $B$ puts the identifier $R_{\text {reply }}$ in the address field of the destination. The sender field of a reply contains the all zero field, to indicate that it is a reply. For every reply $A$ receives, it computes the hash value of $R_{A}, a d d r B$ and the nonce. If this value corresponds to the identifier $R_{\text {reply }}$ in the header of the message, then $A$ knows the message was intended for her. If one would not include $a d d r B$ in the hash, then everybody could have generated the reply. Now $A$ knows that the other device has knowledge of the address of $B$, and probably will be $B$ itself.

An eavesdropper who does not know the address of $B$, is not able to link the reply to the original message. Note that using the identifier $R_{\text {reply }}$ instead of $R_{A}$ avoids tracking. Suppose an attacker performs a replay attack and continuously broadcasts an old message it intercepted (sent from $A$ to $B$ and containing $R_{A}$ in the address field of the sender). If $B$ would put $R_{A}$ in the destination address field of the reply, then this identifier would be reused every time the attacker broadcasts the old message. This way, one can easily detect when device $B$ is present (however, one would not know the identity of $B$ ). As $R_{\text {reply }}$ is based upon a random variable nonce, it will always be different. As a result, an attacker replaying an old message will never see emerging the same identifier and will not be able to detect that $B$ sends a reply to its message.

If $A$ now wants to send a reply back to $B$, it computes the identifier $R_{\text {reply }}$ as shown in eqn. 5:

$$
R_{\text {reply }}=H\left(\operatorname{addr} B, R_{A}, \text { nonce }\right)
$$

Note that this is exactly the same way as shown in eqn. 4 (device $B$ is now the destination and $A$ the sender). $B$ has stored the random identifier $R_{A}$ and can hence detect that the reply comes from $A$ (without knowing the real identity of $A$ ).

We implicitly assume that it is "reasonably safe" to communicate with a device that knows your address, because normally only trusted devices in the WPAN will know this. Note that $a d d r B$ is never transmitted in the clear. It is used as a secret input for the computation of the identifiers $R_{B}$ and $R_{\text {reply }}$.

Attentive readers probably wonder why we do not employ the same solution as in scenario 1 (see Sect. 3.1). At first sight, its looks interesting to use the Bluetooth hardware address of $B$ as secret key to compute a chain of pseudorandom values as follows:

$$
\begin{gathered}
R=P R F_{a d d r B}(I V) \\
R_{\text {new }}=P R F_{\text {addr } B}\left(R_{\text {old }}\right)
\end{gathered}
$$

This would however cause a serious privacy problem. Every time a device starts communicating with $B$ for the first time, it uses the identifier $R$, as shown in eqn. 6. An attacker would detect that $R$ is being reused and hence is able to link the messages to each other. Also the next values in the chain will appear frequently. This problem does not occur in scenario 1 , as $B$ uses a different key for every device it communicates with. Replacing the value $I V$ by a random, variable nonce would solve this linkability problem, but is still not a good idea. The basic problem is that every device that sends a message to $B$, reuses the same secret key $a d d r B$. An active eavesdropper can hence perform a replay attack. Suppose the attacker would continuously broadcast an old message it intercepted (sent from $A$ to $B$ and containing an identifier $R_{1}$ ). If $B$ is in the neighborhood, it would respond with a message embodying the identifier $R_{2}$, as shown in eqn. 8.

$$
R_{2}=P R F_{a d d r B}\left(R_{1}\right)
$$

The next time the attacker broadcast a message containing $R_{1}, B$ will again use the identifier $R_{2}$. This hence enables the attacker to detect the presence of device $B$ (without knowing the exact identity of $B$ ) and accordingly track it. This problem is caused by the fact that only the first input of the chain is variable. Reusing this first input results in the same chain of pseudo-random values. The response of $B$ has to include a variable input. This is however completely equivalent to the solution in scenario 2 , described earlier in this section.

Energy consumption is again taken into account. In our solution, we compute hash values and use the result as an identifier (instead of the Bluetooth hardware address). This technique is very efficient. Suppose one uses the SHA-1 algorithm to calculate the hash values. Experiments show that this hash function consumes $0.76 \mu \mathrm{J} /$ Byte on the Intel SA1110 StrongARM processor [20]. If we assign 48 bits of the payload to the nonce, the cost of computing $R_{B}$ and $R_{\text {reply }}$ is $9.12 \mu \mathrm{J}$ and $13.68 \mu \mathrm{J}$ respectively. This is completely negligible to the cost of transmitting or receiving two 48-bit identifiers (one of the sender and one of the destination), which is $2016 \mu \mathrm{J}$ and $1340 \mu \mathrm{J}$ respectively.

\subsection{Scenario 3: Secure out-of-band channel available}

Mobile devices often share two communication channels: an insecure, high-bandwidth channel (Bluetooth in this case) and an extra, secure, low-bandwidth channel. This extra channel is sometimes also called the out-of-band channel. The situation is conceptually shown in Fig. 2. Typical examples of out-of-band channels are infrared (IrDA [13]), phys- 


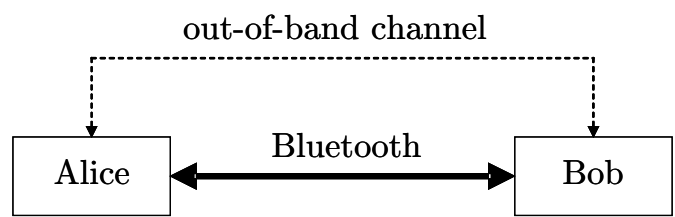

Figure 2: Mobile device share two communication channels

ically connecting both devices [24], the user operating the device, a verbal side channel, ...

Both devices, that do not yet share anything in the beginning of a communication session, will perform a pairing protocol. The goal of such protocol is to establish a highentropy secret key. This will be achieved by exchanging information through both communication channels. One can find several interesting pairing protocols in the literature $[1,10,11,22]$. The choice of which pairing protocol to use, depends on the properties of the available out-of-band channel. Such a channel can be private and/or authentic. When $A$ and $B$ share a private channel, an attacker cannot read messages that are sent via this channel. When $A$ and $B$ share an authentic channel, an attacker cannot modify/delete/insert messages. This way, $A$ knows that only $B$ could have sent the received message (or vice versa). One can also make other classifications: the out-of-band channel can be unidirectional or bidirectional, it can have low or high bandwidth, ...

An easy and practical solution to enable location privacy, is to generate a random identifier and transmit it via outof-band mechanisms or during the pairing protocol. As the former is quite trivial, we will focus on the latter in the rest of this section. By exchanging the random identifier during the execution of the pairing protocol, we realize two goals in the same time: key-establishment and location privacy.

Hoepman shows how a standard Diffie-Hellman protocol can be transformed into a pairing protocol that can be used in an anonymous network [11]. The basic principle of this pairing protocol is as follows. The mobile devices perform the Diffie-Hellman key exchange protocol with each other. The Diffie-Hellman exchange on itself is however vulnerable to a man-in-the-middle attack. That is why the mobile devices will use the authentic out-of-band channel to authenticate the public values that they have sent via Bluetooth. This way, one achieves forward security ${ }^{3}$ [18], which implies that disclosure of the long-term secret keying material that is used to derive a session key does not compromise the secrecy of agreed session keys from earlier runs. The secrecy of the shared key depends on the cryptographic strength of the Diffie-Hellman protocol [7]. The pairing protocols of Hoepman, that can be used in anonymous networks, have the nice property that they do not reveal the identity of the devices performing the pairing protocol.

There are however scenarios where the pairing protocols of Hoepman are not very practical. Suppose that both devices have an input- and/or output-interface and do not share an authentic channel with (relatively) high-bandwidth. The user itself can then be considered as an out-of-band chan-

\footnotetext{
${ }^{3}$ In the literature, perfect forward secrecy is used, but note that perfect secrecy refers to information theoretic security, which is not achieved here.
}

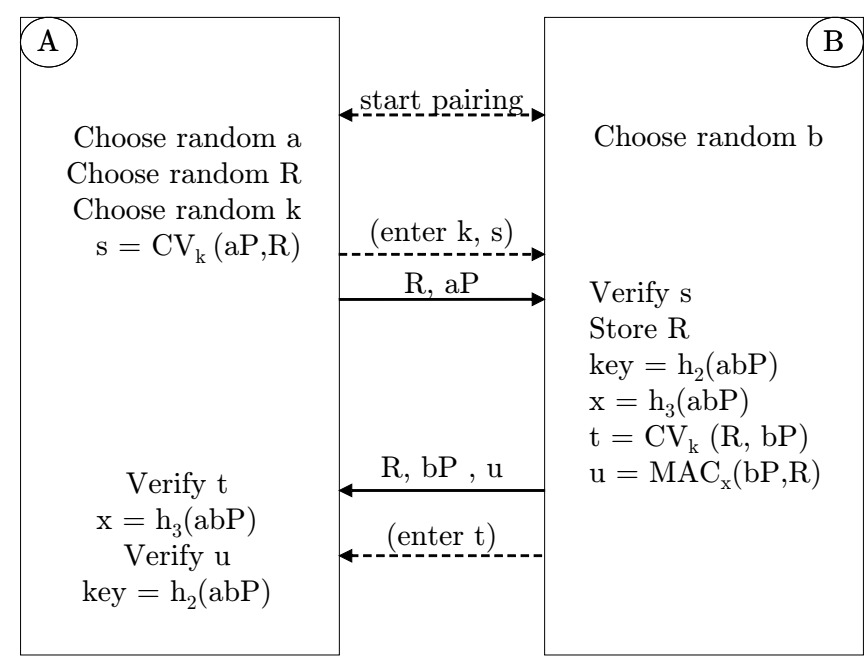

Figure 3: An efficient pairing protocol enabling location privacy

nel, because (s)he can enter values on the input-interface or read whatever is shown on the output-interface of a device. Instead of one user operating both devices, each device can also be operated by its own user and both users then exchange some information (e.g., verbally, since the range of Bluetooth is often only a few meters). In the scenario where the user is the out-of-band channel, one should use pairing protocols in which only a very small amount of bits has to be transferred via the out-of-band channel. Figure 3 shows an efficient pairing protocol that is particularly designed to improve security, without renouncing the user-friendliness [22]. The user only has to enter three small numbers (typically 4 or 5 hexadecimal digits). The basic idea of the protocol is that $A$ and $B$ carry out the Diffie-Hellman protocol in the group of points defined by an elliptic curve $\mathcal{E}$ over a finite field $[17,18]$. When $A$ sends it public key $(a P)$ to $B$, it also transmits a random identifier $R$. At the same time, the user enters three small hexadecimal numbers $(k, s$ and $t$ ) that are used to authenticate both the public key and the identifier. All messages going from $A$ to $B$ (or vice versa), during this pairing protocol, will contain this identifier. An attacker can not observe the information that is transferred via the out-of-band channel and hence does not know that $A$ and $B$ are communicating. Finally, $A$ and $B$ will continue the Diffie-Hellman protocol, but this is out of the scope of this paper. Interested readers are referred to [22] for more details about this advanced pairing protocol. After the execution of the pairing protocol, $A$ and $B$ share a symmetric key and an identifier $R$. To avoid linkability, $R$ has to be updated every time a message is sent. This can be done by employing the techniques described in Sect. 3.1, as both devices now share a symmetric key.

Establishing location privacy during the pairing protocol, is very efficient. If we observe the pairing protocol discussed above, we notice that the devices do not have to perform extra computations. Every operation is already necessary to establish the session key. So we get location privacy for free. The cost of enabling location privacy is memory. $B$ has to store every pair $(a P, R)$ it receives, until $A$ authenticates the correct one. Then $B$ can discard all other pairs. The 
exact amount of data $B$ has to store temporally, depends on how many other devices in the WPAN are performing the pairing protocol in the same time. The scheme is also userfriendly, as a user only has to enter three small hexadecimal numbers. This was one of the design goals of the pairing protocol. The cost of updating the identifier $R$ is already presented in Sect. 3.1.

\subsection{Scenario 4: No shared data available}

In this scenario, we assume that the mobile devices have never communicated before or did not store any information from previous communication rounds (e.g., a session key). The devices also do not know each others' addresses. It is however still possible to enable location privacy.

If a device $(A)$ wants to offer some services, it could generate a random identifier $R_{A}$ and broadcast it during the inquiry phase. When $B$ wants to make use of these services and send a message to $A$, it generates a random identifier $R_{B}$. To construct the header of the message, $B$ puts $R_{A}$ in the address field of the destination and $R_{B}$ in the address field of the sender. These identifiers can then be used for communication between $A$ and $B$. After they finished sending messages to each other, the mobile devices discard both identifiers. If $A$ and $B$ want to start communicating again on a later moment, they both generate a new identifier. Updating the old identifiers is not a secure solution, as both devices do not share any secret information. An attacker could hence perform the same update and keep tracking the devices. Therefore, the devices always generate a new identifier every time they start communicating. The disadvantage of this solution is that a device has absolutely no idea with who it is exchanging messages. This is however also the case in Bluetooth (the way it is currently being used). One has no assurance at all that a device is really the device it claims to be, as it is very easy to spoof a Bluetooth hardware address.

Another solution would be to broadcast every message and not use any identifiers at all. This also enables location privacy, but has the disadvantage that a mobile device has to check the content of every message it receives and decide if the message was intended for him. This can be quite energy consuming. Generating an identifier and transmitting it to other devices does not introduce a significant extra cost, so we recommend to employ this technique.

The trade off between both solutions also depends on the amount of location privacy that is required. By reusing the identifiers $R_{A}$ and $R_{B}$, an attacker is able to link all messages in one communication round. There is however no relation between the temporary identifiers used in different communication rounds, as old identifiers are always discarded after the Bluetooth devices finished sending messages to each other. When the devices start communicating again on a later moment, new random identifiers are generated. If perfect unlinkability is required, then all messages have to be broadcasted. This is significantly more energy consuming, but provides full location privacy. There is hence a trade off between privacy and complexity.

\section{INTEGRATING THE WPAN SCENARIOS}

In the previous section, we demonstrated that location privacy can be enabled in each of the four WPAN scenarios. In the most general use case scenario, all the WPAN scenarios will occur in the same time. A device can share a sym- metric key with some devices, know the Bluetooth hardware address of some other devices, share an out-of-band channel with several devices, and finally share nothing with all other devices in the neighborhood.

Let us again assume that $A$ sends a message to $B$. Sending a message in this general scenario is quite straightforward. After all, $A$ knows exactly what it shares with $B$ and can immediately choose the correct technique to enable location privacy. Receiving a message is less trivial. Pairing (the technique used in scenario 3 ) typically requires userinteraction, that will trigger the execution of the pairing protocol. If the address field of the sender and the destination, in the header of the message, contain the same identifier, then a chain of pseudo-random values is computed to assure location privacy (the technique used in scenario 1 , or in scenario 3 after the execution of the pairing protocol). If both address fields contain a different identifier (distinct from the all zero field), then $B$ checks if the destination address field equals $H(a d d r B$, nonce $)$. If this is the case, then the technique proposed for scenario 2 is employed. Otherwise, the broadcasted message contains two random identifiers (technique used in scenario 4). And finally, if the address field of the sender contains the all zero field, then the technique employed in scenario 2 is used to send a reply to $B$.

After detecting the technique that has been used to enable location privacy, $B$ still does not know which device has sent the message. That is why $B$ has to perform some computations to figure this out. Suppose the technique used in scenario 1 was employed, and that $B$ shares a symmetric key with 10 other Bluetooth devices. Then it has to compute the next value in all 10 of the chains of pseudo-random values it shares with the other devices, and check which value corresponds to the identifier in the received message. Performing such computations is not a problem. Computing the output of a pseudo-random function or a hash function is several orders of magnitude less energy consuming than receiving a message, as shown in the previous section.

The content of the address field of the sender and the destination, in the header of the message, also enables an attacker to make a distinction between several messages. This normally does not cause security problems, unless the network is very dense. If only two devices $(A$ and $B$ ) in the network are communicating, then it is very easy for an attacker to link all the messages to each other. All traffic is going from $A$ to $B$ or vice versa. An attacker can however not link messages from different communication rounds to each other. Perfect unlinkability is hence only assured when there are enough devices in the network that are communicating, to make it impossible for an attacker to perform traffic analysis. Note that untraceability is always assured, even in a dense network.

\section{CONCLUSION}

Location privacy is one of the major security problems in a Wireless Personal Area Network (WPAN). The leakage of the device's unique hardware address enables an attacker to keep track of the place and time a mobile device is communicating. The hardware address of the device can often be linked to the identity of the user operating the mobile device, and this causes severe privacy problems.

While the basic location privacy problem of using a longterm device address can be resolved by using temporary pseudonyms, an incomplete solution can give rise to link- 
ability.

We have presented four WPAN communication scenarios:

- the mobile devices share a symmetric key.

- the mobile devices know each others' addresses.

- a secure extra communication channel is available.

- the mobile devices share nothing.

For each scenario, we have presented several techniques to enable location privacy. We applied low-cost cryptographic primitives to generate a temporary random identifier that can be used to communicate privately. Untraceability is assured in all four WPAN scenarios. Unlinkability can however only be guaranteed if enough mobile devices in the network are communicating in the same time. Our solutions hardly increase the energy consumption. This is important, as mobile devices in a WPAN are typically energy constrained.

One can conclude that the location privacy problem is closely related to the secure initialization of a WPAN by employing pairing protocols. When two mobile devices share secret data, they can easily establish a temporary pseudonym by applying the solutions described in this paper. However, more research is needed to study new and efficient pairing protocols.

\section{Acknowledgments}

Dave Singelée is funded by a research grant of the Institute for the Promotion of Innovation by Science and Technology in Flanders (IWT). This work was also supported by the Concerted Research Action (GOA) Ambiorics 2005/11 of the Flemish Government.

\section{REFERENCES}

[1] D. Balfanz, D. Smetters, P. Stewart, and H. Wong. Talking to Strangers: Authentication in Adhoc Wireless Networks. In Proceedings of the Network and Distributed System Security Symposium (NDSS '02). The Internet Society, 2002.

[2] Bluetags Corporation. http://www.bluetags.com.

[3] Bluetooth Special Interest Group. http://www.bluetooth.com/.

[4] Bluetooth Specification. https://www . bluetooth.org/spec/.

[5] H. Cheung. The Bluesniper Rifle, 2004. http://www . tomsnetworking. com/Sections-article106.php.

[6] DEF CON. Computer Underground Hackers Convention. http://www. def con.org.

[7] W. Diffie and M. Hellman. New Directions in Cryptography. In IEEE Transactions on Information Theory, pages 644-654, 1976.

[8] M. Gruteser and D. Grunwald. Enhancing Location Privacy in Wireless LAN Through Disposable Interface Identifiers: A Quantitative Analysis. In Proceedings of the 1st ACM International Workshop on Wireless Mobile Applications and Services on WLAN Hotspots (WMASH '03), pages 46-55. ACM Press, 2003.

[9] J. Haartsen, M. Naghshineh, J. Inouye, O. Joeressen, and W. Allen. Bluetooth: Visions, Goals and Architecture. In ACM Mobile Computing and Communications Review, pages 38-45, 1998.
[10] J. H. Hoepman. The Ephemeral Pairing Problem. In Financial Cryptography, Lecture Notes in Computer Science, LNCS 3110, pages 212-226. Springer-Verlag, 2004.

[11] J. H. Hoepman. Ephemeral Pairing on Anonymous Networks. In Proceedings of the Second International Conference on Security in Pervasive Computing (SPC 05), Lecture Notes in Computer Science, LNCS 3450, pages 101-116. Springer-Verlag, 2005.

[12] IEEE 802.15, the Wireless Personal Area Network Working Group. http://www. ieee802.org/15/.

[13] Infrared Data Association. http://www.irda.org/.

[14] M. Jakobsson and S. Wetzel. Security Weaknesses in Bluetooth. In Proceedings of the Cryptographer's Track at the RSA Conference (CT-RSA '01), Lecture Notes in Computer Science, LNCS 2020, pages 176-191. Springer-Verlag, 2001.

[15] Loca Project. http://www.loca-lab.org.

[16] A. Lysyanskaya, R. Rivest, A. Sahai, and S. Wolf. Pseudonym Systems. In Proceedings of the 6th Annual International Workshop of Selected Areas in Cryptography (SAC '99), Lecture Notes in Computer Science, LNCS 1758, pages 184-199. Springer-Verlag, 1999.

[17] A. J. Menezes. Elliptic Curve Public Key Cryptosystems. Springer, July 1993.

[18] A. J. Menezes, P. C. van Oorschot, and S. A. Vanstone. Handbook of Applied Cryptography. CRC Press, October 1996.

[19] New European Schemes for Signatures, Integrity, and Encryption. http://www. cryptonessie.org.

[20] N. Potlapally, S. Ravi, A. Raghunathan, and N. Jha. Analyzing the Energy Consumption of Security Protocols. In Proceedings of the 2003 International Symposium on Low Power Electronics and Design (ISLPED '03), pages 30-35. ACM Press, 2003.

[21] A. Shamir. Identity-Based Cryptosystems and Signature Schemes. In Advances in Cryptology CRYPTO '84, Lecture Notes in Computer Science, LNCS 196, pages 47-53. Springer-Verlag, 1984.

[22] D. Singelée and B. Preneel. Improved Pairing Protocol for Bluetooth. To Appear in the Proceedings of the 5 th International Conference on Ad-Hoc, Mobile, and Wireless Networks (ADHOC-NOW'06).

[23] D. Singelée and B. Preneel. Review of the Bluetooth Security Architecture. Information Security Bulletin, 11(2):45-53, 2006.

[24] F. Stajano and R. Anderson. The Resurrecting Duckling: Security Issues in Ad-Hoc Wireless Networks. In Proceedings of the 7th International Workshop on Security Protocols, Lecture Notes in Computer Science, LNCS 1796, pages 172-182. Springer-Verlag, 1999.

[25] The WI-FI alliance. http://www.wi-fi.org/.

[26] F. Wong and F. Stajano. Location Privacy in Bluetooth. In Proceedings of 2nd European Workshop on Security and Privacy in Ad hoc and Sensor Networks (ESAS '05), Lecture Notes in Computer Science, LNCS 3813, pages 176-188. Springer-Verlag, 2005 . 